\title{
Stability of microencapsulated spray drying carotenoids depending on the composition of the encapsulating matrix
}

\begin{abstract}
Carotenoids are bioactive compounds that can be found in different food matrices. However, these compounds are easily degradable at high temperatures, light and oxygen. Therefore, it is necessary to search for an alternative that avoids such deterioration. Objective: The present study aimed at evaluating the storage stability of microencapsulated palm (Elaeis guineensis) and urucum (Bixa orellana) oil carotenoids by spray drying, depending on the composition of the encapsulation matrix. Methodology: a comparison was made between two types of formulations, a layer composed of isolated soy protein and a double layer composed of isolated soy protein and pectin. The procedure was split into three stages: 1) formulation of stable emulsions; 2) spray drying and 3) characterization of the microcapsules by evaluation of parameters such as encapsulation efficiency, color, humidity and stability of carotenoids over a period of 45 days. Conclusion: the addition of pectin as a double-layer encapsulation material is favorable for all the evaluated parameters except humidity, for which it was not possible to analyze its response during drying.
\end{abstract}

Keywords: microencapsulation, spray drying, carotenoids
Volume 7 Issue 2 - 2020

\author{
Hernandez-Chamorro Tatiana,' Vânia Regina \\ Nicoletti Telis, ${ }^{2}$ Poliana Moser, ${ }^{2}$ Benavides \\ Iván Felipe ${ }^{3}$ \\ 'Faculty of Engineering, Universidad de Caldas, Colombia \\ ${ }^{2}$ Department of Food Engineering and Technology, Universidad e \\ Estadual Paulista, Brazil \\ ${ }^{3}$ Grupo de Investigación Arena, Universidad de Nariño, \\ Colombia
}

\begin{abstract}
Correspondence: Ivan Felipe Benavides, Group of Investigation SAND, Universidad de Nariño, Colombia, Email pipeben@gmail.com
\end{abstract}

Received: February 06, 2020 | Published: April 09, 2020

\section{Introduction}

This research focuses on studying the influence of pectin as a wall material for the microencapsulation of oils rich in carotenoids by using the spray drying technique. The basic materials for this research were oils from Palm (Elaeis guineensis) and Urucum (Bixa orellana), for which literature report high levels of carotenoids.Besides the various applicaitons of palm oil for food industry, it is also useful for making soaps, candles, detergents, pharmaceuticals, cosmetics and natural dyes. ${ }^{1,2}$ This oil is considered as an important source of antioxidants, topherols, tocotrienols, carotenoids, phytosterols, phenolic compounds and other phyto-nutrients. ${ }^{2}$ Urucum peas are used to extract a pigment used for human and animal feeding. ${ }^{3-5}$ Urucum seeds has two colorants, the bixin (red), oil-soluble, representing more than $80 \%$ of the total carotenoids, and the norbixin (yellow), water-soluble. ${ }^{4,6}$ Microencapsulation is defined as the process by which the encapsulating material covers small particles of a target compound (active material), which is integrated into a homogeneous or heterogeneous matrix that creates a physical barrier between the active material and the environment. ${ }^{7-9}$ Spray drying is an unitary operation that aims to transform a fluid-state raw material to dry particles. ${ }^{10}$

This is one of the most complex multiphaseconvection drying processes that involves different stages such as particle atomization, bedding transport, evaporation and others. ${ }^{8}$ This operation produces high quality powders, low water activity and good storage capacity, and is one of the most used operations for drying emulsions and suspensions that contains oils and flavors. ${ }^{11,12}$

During spray drying microencapsulation process, the wall system is designed to protect the core material from factors causing deterioration, prevent the premature interaction between the core material and other ingredients and limit the volatile losses ${ }^{13} \mathrm{Criteria}$ to select a wall material mainly rely on physical-chemical properties such as solubility, molecular weight, glass transition temperature, crystallinity, diffusivity, layer formation and emulsifyingproperties. ${ }^{7}$ For oils, and efficient microencapsulation requires a encapsulation material with a highemulsifying activity, a high sensitivity, a tendency to form a thin and dense wall during drying, and it must not allow the separation of lipids from the emulsion during dehydration. ${ }^{13-15}$ Biopolymers, proteins and maltodextrins from different sources are frequently used for microencapsulation (e.g, arabic gum, alginates, carrageenan, milkproteins, jelly, dextrose and waxes). ${ }^{16,17}$ The objective of this research was to study the microencapsulation process of richcarotenoid oils by spray drying, when using different formulations of wall materials (one andtwo layers). Furthermore, it aimed at investigating the effect of these formulations on the stability of carotenoids during the storage of microcapsules.

\section{Materials and methods}

\section{Stage I: Formulation of stable emulsions}

Emulsions of oil in water were composed by oil, soy protein isolates (SPI), pectin (PEC) and maltodextrin (MDX). PEC and MDX had variable formulations of emulsions. Treatments for PEC factor were a control no-PEC addition (formulations A-C) and PEC addition with $0.9 \mathrm{~g}$ (formulations B-D) (Table 1). An ultra-turrax stirrer (T-25 IKA Germany) was used for the formulation of emulsions at 1400 rpm. The oil was dispersed over the SPI and shaken during 4 minutes. The high-methoxylation PEC solution was added to the SPI and shaken during 3 minutes. Finally MTX was added and shaken during 2 minutes. The resulting emulsion was subjected tosonication $(30 \mathrm{~Hz}$ frequency) during 1 minute according to the methods proposed by Kaltsa et al. ${ }^{18}$ with some modifications.

\section{Stage 2: Spray drying}

A spray drying bench (B-290, Buchi Suiza) was used for microencapsulation. This was equipped with a dual fluid spray nozzle 
and a peristaltic pump for microcapsule input. Parameters for drying were: input flow of $3 \mathrm{ml} / \mathrm{min}$, atomization air flow of $500 \mathrm{l} / \mathrm{h}$, air input temperature of $180^{\circ} \mathrm{C}$ and suction of $90 \%$.

\section{Stage 3: Characterization of microcapsules}

Response variables to evaluate the effect of PEC addition on microcapsules were color, humidity, drying performance, carotenoid stability and microencapsulation efficiency. Color measurements were performed in a colorimeter (CR-S Konica Minolta, Japan) by extracting the luminosity parameter (L) and chromatic coordinates a (+a: red and -a: green) and b (+b:yellow and -b:blue). These parameters were used to calculate chroma (C) and pitch angle(h) according to Konica Minolta. ${ }^{19}$ Humidity of microcapsules were gravimetrically measured by drying $1.5 \mathrm{~g}$ of sample in a vacuum over at $70^{\circ} \mathrm{C}$ and constant weight. This process was repeated three times according to AOAC 20. Drying performance was determined by the relationship between the solid mass in the powder (dryer output) and the solid mass in the feeding solution of the dryer (dryer input).
Microencapsulation efficiency was determined by the relationship between the amount of non-encapsulated oil on the surface (Os) and the total amount of oil in the formulation $(\mathrm{Ot})$. Os was determined according to the method described by Tono et al., ${ }^{21}$ with minor modifications. $15 \mathrm{ml}$ of hexane were added to $1.5 \mathrm{~g}$ of microcapsule powder in a covered

glass. This mixture was shaken during 2 minutes at $25^{\circ} \mathrm{C}$ and filtered through a What mean No1 paper filter. The resulting powder was washed three times with $20 \mathrm{ml}$ of hexane. The filtered solution was allowed to evaporate at $25^{\circ} \mathrm{C}$ until constant weight. Finally, Os was determined by mass difference.Water content analysis and carotenoid quantification were performed to determine the stability of microencapsulated carotenoids. To achieve this, microcapsule samples were distributed in petri boxes during 45 days and the analysis were performed every seven days. Storage conditions were temperature of $35^{\circ} \mathrm{C}$ and relative humidity of $34 \% . \mathrm{k}$ Carotenoid analysis and humidity quantification were performed according to Tono et al. ${ }^{21}$ Water content was determined gravimetrically according to AOAC 20 by drying in a vacuum oven at $60^{\circ} \mathrm{C}$ until constant weight..$^{23}$

Table I Formulation of emulsions of palm oil and urucum oleoresin with varying wall materials ( $100 \mathrm{~g}$ sample)

\begin{tabular}{llllll}
\hline Sample & Oil (g) & MDX (g) & PEC (g) & SPI (g) & $\begin{array}{l}\text { Distilled } \\
\text { water (g) }\end{array}$ \\
\hline A (urucum oleoresin) & 10 & 34 & 0 & 2 & 54 \\
B (urucum oleoresin) & 10 & 33,1 & 0,9 & 2 & 54 \\
C (Palm oil) & 10 & 34 & 0 & 2 & 54 \\
D (Palm oil) & 10 & 33,1 & 0,9 & 2 & 54 \\
\hline
\end{tabular}

\section{Statistical analysis}

The results were analyzed with simple Analysis of Variance (ANOVA) and post-hoc pairwise Tukey comparisons. Colorimetry, humidity, drying performance, carotenoid stability an microencapsulation efficiency were considered as dependent variables as a function of factors PEC and time.Significance level was set to 0.05 and confidence level to $95 \%$ R-studio 21 software was used for the analyzes.

\section{Results and discussion}

\section{Colorimetry}

As shown in Table 2, data showed significant differences for all the evaluated colorimetry variables. Samples A and C (no-PEC) showed less clarity and higher values for chromatic coordinates $\mathrm{b}^{*}$, which suggests a higher yellow intensity. Theses results suggest that PEC addition produces a darker color in microcapsules and generates a double layer wall that helps to conserve the oil carotenoids by protecting them from light. Most of the microcapsules showed a significant difference for $\mathrm{h}$ (p\&lt;0.05). However, variance among microcapsules were less than two degrees, suggesting a minimum difference indicating yellow hues (Figure 1). Humidity, drying performance, encapsulation efficiency and carotenoid retention. Data shown in table 3 did not provide relevant information to guarantee that microcapsules with added PEC increase water losses during the drying process. Water loss was higher for palm oil and lower for urucum oleoresin. Therefore, more research is needed to achieve a better conclusion about this phenomenon. However, it is important to consider that humidity levels were statistically similar among samples without PEC addition (A and C).

Drying performance was statistically different between samples with different wall materials. As expected, formulations with PEC addition ( $\mathrm{B}$ and $\mathrm{D}$ ) showed higher drying performances, suggesting that PEC facilitates the spray drying process. These results indicate that PEC helps MXT to easily adhere to the microcapsules which were previously formed by SPI. As shown in table 3, PEC addition produced a higher encapsulation efficiency. PEC helps

microcapsules to prevent their collapse due to temperature and pressure during spray drying. As shown in table 3 , microcapsules with PEC (B and D) had a higher carotenoid retention, suggesting a lower exposition of oils to high temperatures during spray drying. Hence, the function of PEC as a component of the encapsulating matrix was as expected.

\section{Stability of microencapsulated carotenoids during storage}

As shown in figures $2 \mathrm{~A}$ and $2 \mathrm{~B}$, the gain of humidity across days was significantly higher in $\mathrm{A}$ and $\mathrm{C}$. This means that PEC addition helps to prevent humidity gains in microcapsules across time. The dates show a considerable deterioration of these bioactive compounds for all the formulations, specially during the first three weeks of storing. However, it was also evident that the losses of these components in PEC-added microcapsules were lower for both palm oil and urucum oleoresin. 
Table 2 Color variable results clarity $\left(\mathrm{L}^{*}\right)$, chromatic coordinates ( $\mathrm{a}^{*}$ and $\left.\mathrm{b}^{*}\right)$, Chroma $(\mathrm{C})$ and Hue angle $(\mathrm{h})$ of the microcapsules

\begin{tabular}{llllll}
\hline Sample & $L^{*}$ & $a^{*}$ & $b^{*}$ & $C$ & $h$ \\
\hline A & $84,54(0,50)^{\mathrm{c}}$ & $10,26(0,04)^{\mathrm{b}}$ & $38,89(0,05)^{\mathrm{a}}$ & $40,22(0,052)^{\mathrm{a}}$ & $75,22(0,04)^{\mathrm{c}}$ \\
B & $87,08(0,19)^{\mathrm{b}}$ & $8,67(0,0,05)^{\mathrm{e}}$ & $30,21(0,03)^{\mathrm{b}}$ & $31,45(0,017)^{\mathrm{b}}$ & $73,99(0,10)^{\mathrm{d}}$ \\
C & $84,47(0,52)^{\mathrm{d}}$ & $9,4 I(0,06)^{\mathrm{d}}$ & $60,00(0,6 \mathrm{I})^{\mathrm{c}}$ & $60,74(0,598)^{\mathrm{c}}$ & $81,09(0,14)^{\mathrm{e}}$ \\
D & $84,78(0,37)^{\mathrm{c}}$ & $9,65(0,06)^{\mathrm{d}}$ & $63,89(0,47)^{\mathrm{d}}$ & $64,6 \mathrm{I}(0,387)^{\mathrm{d}}$ & $8 \mathrm{I}, 4 \mathrm{I}(0,04)^{\mathrm{e}}$
\end{tabular}

Table 3 Humidity, Performance, Encapsulation efficiency, Carotenoidretention

\begin{tabular}{lllll}
\hline Sample & Humidity (\%) & Performance (\%) & $\begin{array}{l}\text { Encapsulation } \\
\text { efficiency (\%p/p) }\end{array}$ & Carotenoidretention (\%) \\
\hline A & $7,130(0,141)^{\mathrm{a}}$ & 49,519 & $23,94(3,32)^{\mathrm{b}}$ & $38,267(0,66)^{\mathrm{c}}$ \\
B & $9,528(0,344)^{\mathrm{b}}$ & 53,757 & $37,16(2,02)^{\mathrm{d}}$ & $47,854(0,83)^{\mathrm{d}}$ \\
C & $6,1760,606)^{\mathrm{a}}$ & 37,917 & $23,62(3,14)^{\mathrm{b}}$ & $57,240(0,672)^{\mathrm{e}}$ \\
D & $4,642(0,344)^{\mathrm{c}}$ & 49,216 & $46,87(4,1 \mathrm{I})^{\mathrm{f}}$ & $67,947(0,43)^{\mathrm{f}}$ \\
\hline
\end{tabular}

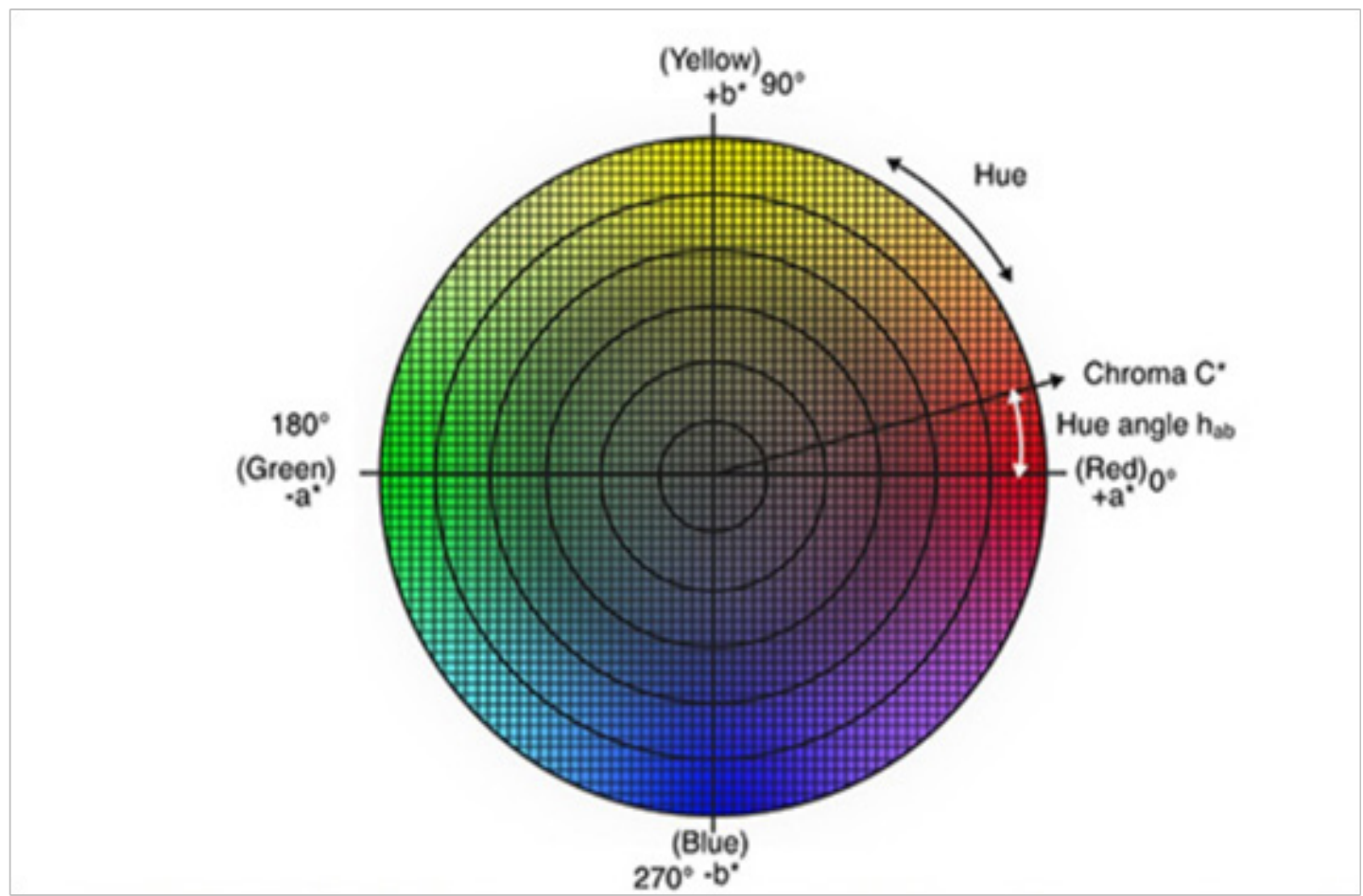

Figure ICIE XYZ, CIE *a *b *c color spaces including CIE *c*h used for communicate and expressthe color of objects. ${ }^{19}$ 


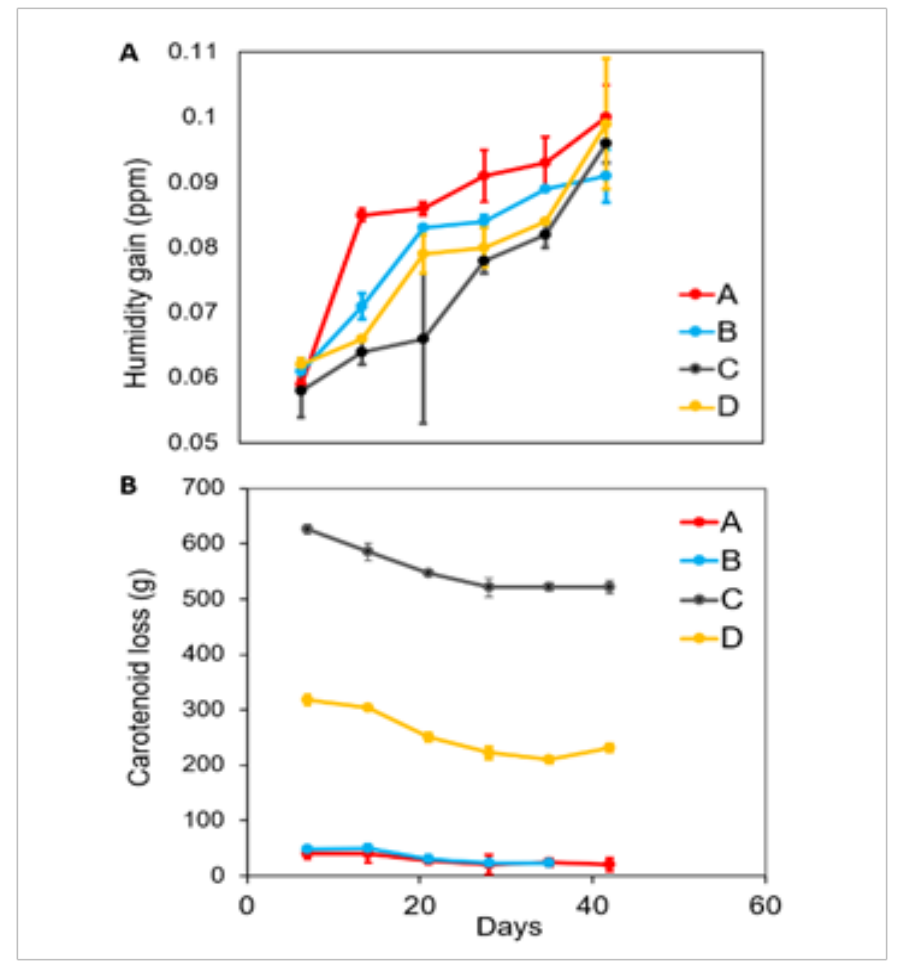

Figure 2 A) Humidity gain of formulations A, B, C and D across storage time, B) Carotenoid loss offormulations A, B, C and D acrossstorage time. Vertical bars represent 2 standard deviations.

\section{Conclusions}

Using PEC as a second component of microcapsule walls helped to a considerable increase in drying performance and microencapsulation efficiency. This increased the conservation of carotenoids. PEC addition did not affected the perception of yellow hues typical in oils. The behaviour of sample with double wall material during the 45-days storage period was favourable for all the evaluated response variables. Using PEC as an encapsulating material decreased the gain of humidity and conserved carotenoids with higher efficiency than non-PEC microcapsules during all the storage period.

\section{Acknowledgments}

None.

\section{Conflicts of interest}

None.

\section{Funding}

None.

\section{References}

1. Teixeira CB, Macedo GA, Macedo JA, et al. Simultaneous extraction of oil and antioxidant compounds from oil palm fruit (Elaeis guineensis) by an aqueous enzymatic process. Bioresource Technology. 2013;129:575581.

2. Choo Yuen May. Carotenoids from palm oil. Palmas Magazine. 1996;17(1):71-76.

3. Dem DO. Palm oil: Biochemical, physiological, nutritional, hematological, and toxicological aspects: A review. Plant Foods for Human Nutrition. 2002;57:319-341.
4. de Melo Braz, Nádia. Residual annatto seed in commercial laying hens: performance and egg characteristics. Acta Scientiarum. 2007;29:2.

5. Castro CB de. The culture of annatto. Brasília, DF: EMBRAPA; 1994.

6. Demczuk Jr, Bogdan Ribani, Rosemary Hoffmann. News about the chemistry and use of annatto (Bixa orellana L.). Brazilian Journal of Food Research. 2015;6(1):37-50.

7. Manke Nachtigall Aline. Saponification study in annatto pigments. Food Science and Technology. 2009;29:4.

8. Gharsallaoui A, Roudaut G, Chambin O, et al. Applications of spraydrying in microencapsulation of food ingredients: An overview. Food Research International. 2007;40(9):1107-1121.

9. Schmitz-Schug I, Kulozik U, Foerst P. Modeling spray drying of dairy products -Impact of drying kinetics, reaction kinetics and spray drying conditions on lysine loss. Chemical Engineering Science. 2016;141:315-329.

10. Aranha CPM. Microencapsulation by ionic gelation and electrostatic interaction of the buriti dye (Mauritia flexuosa L. f.). Paulista State University “Julio de Mesquita Filho”, São José do Rio Preto. 2014.

11. Keshani S, Daud WRW, Nourouzi MM, et al. Spray drying: An overview on wall deposition, process and modeling. Journal of Food Engineering. 2015;146:152-162.

12. Watanabe Y, Fang X, Adachi S, et al. Oxidation of 6-O- arachidonoyl 1-ascorbate microencapsulated with a polysaccharide by spray-drying. LWT-Food Science and Technology. 2004;37(4):395-400.

13. Belén-Camacho DR, Moreno-Álvarez MJ, Alemán R, et al. Fruits effect of drying temperature on carotenoid degradation in rhubarb fruits (Jessenia polycarpa Karst). CYTA-Journal of Food. 2004;4(3):206-210.

14. Shahidi F, Han XQ. Encapsulation of food ingredients. Critical Reviews in Food Science \&amp; Nutrition. 1993;33(6):501-547.

15. Abirached Cecilia, Medrano Alejandra, Panizzolo Luis Alberto, et al. Destabilization of oil-water emulsions prepared with soy proteins. Innotec (6 ene-dic). 2011;07:10.

16. Shamaei S, Seiiedlou SS, Aghbashlo M, et al. Microencapsulation of walnut oil by spray drying: Effects of wall material and drying conditions on physicochemical properties of microcapsules. Innovative Food Science \&amp; Emerging Technologies. 2017;39:101-112.

17. Reineccius GA, Ward FM, Whorton C, et al. Developments in gum acacias for the encapsulation of flavors: ACS Publications. 1995.

18. Drusch Stephan. Sugar beet pectin: A novel emulsifying wall component for microencapsulation of lipophilic food ingredients by spray-drying. Food Hydrocolloids. 2007;21(7):1223-1228.

19. Kaltsa O, Michon C, Yanniotis S, et al. Ultrasonic energy input influence on the production of sub-micron $\mathrm{o} / \mathrm{w}$ emulsions containing whey protein and common stabilizers. Ultrasonics sonochemistry. 2013;20(3):881-891.

20. Konica Minolta. Understanding the CIE L * C * h Color Space. 1997.

21. Tonon RV, Grosso Carlos CRF, Hubinger MD. Influence of emulsion composition and inlet air temperature on the microencapsulation of flaxseed oil by spray drying. Food Research International. 2011;44(1):282-289.

22. Fred J Baur, Luther G Ensminger. Official methods of analysis of the Association of Official Analytical Chemists. AOAC. 1990.

23. R-Studio Team. RStudio: Integrated Development for R. RStudio, Inc., Boston, MA. 2017. 